Culture et histoire dans l'espace roman

4 | 2010

Les représentations du corps dans la litterature latinoaméricaine

\title{
Performance du genre et métamorphoses du corps dans les Caraïbes
}

(L. Padura Fuentes, P. J. Gutiérrez et D. Torres)

\section{Nicolas Balutet}

\section{(2) OpenEdition Journals}

Édition électronique

URL : https://journals.openedition.org/cher/8625

DOI : $10.4000 /$ cher.8625

ISSN : 2803-5992

\section{Éditeur}

Presses universitaires de Strasbourg

\section{Édition imprimée}

Date de publication : 30 juin 2010

Pagination : 27-37

ISBN : 978-2-35410-007-0

ISSN : 1968-035X

\section{Référence électronique}

Nicolas Balutet, «Performance du genre et métamorphoses du corps dans les Caraïbes », reCHERches [En ligne], 4 | 2010, mis en ligne le 15 décembre 2021, consulté le 25 janvier 2022. URL : http:// journals.openedition.org/cher/8625; DOI : https://doi.org/10.4000/cher.8625 


\title{
Performance du genre et métamorphoses du corps dans les Caraïbes (L. Padura Fuentes, P. J. Gutiérrez et D. Torres)
}

\author{
Nicolas Balutet \\ Université « Jean Moulin » - Lyon 3
}

\begin{abstract}
Séraphîtüs ôta sa pelisse fourrée de martre, s'y roula et dormit. Le vieux serviteur resta pendant quelques moments debout à contempler avec amour l'être singulier qui reposait sous ses yeux, et dont le genre eût été difficilement défini par qui que ce soit, même par les savants.
\end{abstract}

Honoré de Balzac [Séraphîta, 1834]

Il arrive souvent que le sexe de l'âme ne soit point pareil à celui du corps, et c'est une contradiction qui ne peut manquer de produire beaucoup de désordres.

Théophile Gautier [Mademoiselle de Maupin, 1835]

En tout être humain survient une vacillation d'un sexe à l'autre et, souvent, seuls les vêtements maintiennent l'apparence masculine ou féminine, tandis qu'en profondeur le sexe contredit totalement ce qui se laisse voir en surface.

Virginia Woolf [Orlando, 1928]

\section{Pour Héctor, Jerimy et Eric}

F n 1981, Michel Sardou se demandait ce qu'était «être une femme» et en donnait une vision qui témoigne, à mon sens, du machisme et de la misogynie de l'artiste et de Pierre Delanoë, le co-parolier. Femme de pouvoir, séductrice, manipulatrice, régnant grâce à son corps, la femme des années quatre-vingt de Michel Sardou confirme en creux la peur masculine de perdre le pouvoir. Je n'apprendrai rien à personne en disant que les femmes ont été, 
au cours de l'histoire des hommes et à de rares exceptions près, dévalorisées et assujetties à leur pendant masculin. J'ai rapporté ailleurs les raisons qui peuvent expliquer ce terrible constat (Balutet 2004: 77-88; 2008: 105-107). $\mathrm{Si}$, pour Michel Sardou, "être une femme» est "un étrange drame», il est pourtant impossible de définir l'essence de la femme, comme celle de l'homme d'ailleurs. On tente encore de nous faire croire - les magazines féminins en premier lieu - qu'être une femme c'est, à la fois, être belle, douce, attentionnée, réceptive, épouse, mère et, en même temps, égaler les qualités masculines que l'on continue d'associer au fait d'être dur et ambitieux ${ }^{1}$. Ces explications biologiques et naturalistes qui associent la femme à la nature et l'homme à la culture ont été mises à mal depuis longtemps déjà par Sherry Ortner (1972: 5-31) notamment. Dans les années 90, Judith Butler, Marjorie Garber et d'autres ont bien montré qu'aucun instinct ne pousse la femme (et l'homme) à agir d'une façon plutôt que d'une autre, rien de «naturel» ne la pousse à adopter telle ou telle attitude ou à endosser tel ou tel costume. Ce sont « des inventions fabriquées et maintenues grâce à des signes corporels et à d'autres moyens discursifs» (Garber 1992: 134). L'anthropologue italien, Franco La Cecla (2002: 161-162), n'affirme pas autre chose pour les hommes dans un ouvrage récent qui se veut le pendant masculin du Deuxième sexe de Simone de Beauvoir:

Grandir comme un petit garçon signifie apprendre, par des signes extérieurs et intérieurs, que quelque chose se développe qui prend corps et se forme, qui, sous le regard d'autres hommes ou d'autres femmes, devient un corps masculin, un corps qui est fait par moi autant que par le regard, la voix ou les allusions d'autrui [...]. Ils me font homme artificiellement - et dans certains endroits, douloureusement, par une circoncision - en me faisant porter un pantalon court, une petite cravate, mon premier "complet», une vraie cravate; je deviens homme, avec le nœud des chaussures, le duvet sur le visage et sur le torse. Et j'interprète, je devine par ces signes naturellement artificiels qu'en moi, il y a un fait qui prend forme, un «destin » que je devrai suivre longtemps - en me cherchant, en me trouvant embarrassé, en me perdant, en me regardant dans le miroir, en demandant autour de moi, en me rasant pour la première fois.

1 À la question "Comment construit-on, chez nous, le masculin?», le sociologue Daniel Welzer-Lang (2004: 297) reconnaît que: «Être homme, c'est être le maître chez soi. Être homme, c'est ne pas afficher sa sensibilité, ne pas pleurer. Être homme, c'est être un valeureux guerrier, en lutte permanente pour être le meilleur, le plus performant. Être homme, c'est défendre les pauvres, la veuve et l'orphelin. Bref, être homme, c'est ne pas être une femme. Se distinguer de ces êtres faibles que justement l'homme - le vrai - doit savoir dominer et protéger». 
En ce sens, on comprend pourquoi les féministes ont souvent critiqué les travestis masculins qui produisent, selon elles, une image unifiée, parodique et dégradante de la femme.

À travers l'étude de trois romans d'auteurs caribéens (Leonardo Padura Fuentes, Pedro Juan Gutiérrez, Daniel Torres), je vais m'intéresser précisément aux figures du travesti et du transsexuel ${ }^{2}$ pour essayer de mettre en lumière les valeurs subversives ou, au contraire, conservatrices qu'elles véhiculent. Si le travesti ne doit pas être confondu avec le transsexuel, tous deux partagent des points communs. Un travesti ou drag dans le monde anglo-saxon est une personne qui s'habille avec des vêtements du genre opposé indépendamment de l'identité et de l'orientation sexuelle, l'adepte du travestissement pouvant être hétérosexuel. Il utilise des artifices allant des rembourrages divers aux prothèses amovibles pour se rapprocher de l'esthétique recherchée sans transformations chirurgicales ou médicales. $\mathrm{Si}$ les personnes exprimant une identité travestie ne vivent pas de manière conflictuelle le genre qui leur a été attribué à leur naissance, elles éprouvent cependant le besoin de porter des vêtements et d'autres signes distinctifs considérés socialement comme étant inappropriés pour leur sexe. On peut être travesti et ne pas ressentir de volonté de changer de sexe. Un transsexuel est, en revanche, une personne dont l'identité de genre (sexe psychologique) est en opposition radicale avec ce qu'indique son sexe (anatomique). Cette personne a donc recours à des traitements hormonaux ou chirurgicaux visant à mettre en harmonie son corps et son identité.

Dans Conversaciones con Aurelia (Torres 2007), deuxième volet d'une trilogie incluant Morirás si da una primavera (1993) et Lucy, tú que sabes (en cours d'écriture), l'écrivain portoricain Daniel Torres ${ }^{3}$ nous plonge dans l'univers confiné des cabarets de travestis, un monde que l'auteur a approché en se rendant à de nombreux shows et en recueillant les témoignages de travestis dans les coulisses. Il en ressort, conformément aux critiques émises par les féministes, que le travesti masculin peut adopter, face à la femme, une attitude conservatrice dans la mesure où son «essence» n'est exhalée que par le port d'un certain nombre d'attributs et de vêtements stéréotypés. L'un des drags, Delirio, arbore ainsi des «tacas negras de charol», un «traje rojo de

2 J'utilise ici les termes masculins dans la mesure où je ne vais aborder que le passage d'homme à femme (Mtf).

3 Daniel Torres (1961) est actuellement professeur d'espagnol à Ohio University. Spécialiste de poésie latino-américaine coloniale et contemporaine, on lui doit plusieurs essais, recueils de poésie dont Fusilado Dios (2000) et Invasión de ternura (2004), et romans parmi lesquels Morirás si da una primavera (1993) et Conversaciones con Aurelia (2007). 
lentejuelas nacaradas», « un boa negro» (16), une goutte de Chanel n ${ }^{\circ} 5$ (71), ce parfum dont Coco Chanel disait qu'il «sent la femme», de longs faux-cils, une perruque, le corps épilé (20), etc. Dans El Rey de la Habana (Gutiérrez 1999) de Pedro Juan Gutiérrez ${ }^{4}$, le personnage de Sandra, qualifié par le protagoniste de travesti, arbore de même une perruque blonde, une couleur qui exsude la féminité (62/79), un mini-short noir, une blouse blanche en satin brillant et des talons hauts (79). Quant à Alexis dans Máscaras (Padura Fuentes 1997)5, tout de rouge vêtu, il porte une longue robe, un châle sur les épaules et une ceinture de soie (34). En résumé, les féministes critiquent, à juste titre, l'idée que l'auteur argentin Copi a résumée ainsi: «ser mujer es solamente eso: vestirse de mujer» (Tcherkaski 1998: 50).

Le travesti peut cependant apparaître beaucoup plus subversif. Dans Trouble dans le genre. Le féminisme et la subversion de l'identité (Butler 2006), Judith Butler, après avoir dévoilé le pouvoir répressif de la norme hétérosexuelle, propose une conceptualisation du genre comme performance. Pour elle, "être un homme» ou "être une femme» consiste à réaliser des performances de la masculinité et de la féminité. La figure du travesti soutient cette théorie que la féminité n'a rien d'inné mais qu'elle est, au contraire, une construction culturelle normalisée qui peut donc être jouée, interprétée et imitée: «en imitant le genre, le travestissement révèle implicitement la structure imitative du genre en lui-même, de même que sa contingence» nous explique ainsi Judith Butler (2006: 261). On retrouve chez les deux auteurs cubains de fréquentes allusions à l'idée d'imitation que propose le travesti à travers les références au déguisement (Padura Fuentes 1997: 49/53/91; Gutiérrez 1999: 62), au Carnaval (Padura Fuentes 1997: 32) ou au théâtre (Padura Fuentes 1997: 89), mais c'est le texte de Padura Fuentes qui illustre le mieux les propos de Butler. Grâce aux explications de El Recio et du Marquis, le détective Mario Conde comprend que le travesti transcende les limites du genre et l'exprime à l'aide d'une métaphore picturale:

4 Pedro Juan Gutiérrez (1950) est le maître du «réalisme sale» à la cubaine. Fidèle élève de Bukowski et Henry Miller, on lui doit trois livres de nouvelles (Anclado en tierra de nadie, Nada que hacer et Sabor a mí) qui forment la Trilogía sucia de la Habana (1998) ainsi que plusieurs romans dont Animal tropical (2000) et El Rey de la Habana (1999). Ce dernier conte les déambulations dans les rues de La Havane d'un adolescent, Rey, et sa rencontre avec un monde marginal et crépusculaire.

5 Máscaras (1997) de l'auteur cubain Leonardo Padura Fuentes (1955) est le troisième opus de la tétralogie Las cuatro estaciones avec Pasado Perfecto, Vientos de Cuaresma et Paisaje de otoño. L'action, qui se déroule durant l'été 1989, tourne autour du meurtre d'Alexis Arayán Rodríguez découvert étranglé et travesti en femme. 
como la mariposa, puede pintarse a sí mismo, hacer de su cuerpo el soporte de su obra máxima, convertir sus emanaciones sexuales en color, a través de los aturdidores arabescos y los tintes incandescentes de un ornamento físico. (Padura Fuentes 1997: 100).

Par ailleurs, le choix de la femme imitée dans les cabarets peut apparaître lui aussi subversif dans la mesure où il s'agit toujours d'une femme qui rompt le carcan dans lequel la société voudrait l'enfermer. Dans le roman de Daniel Torres, qu'ont en commun Lucy Fabery, Sara Montiel, Isabel Pantoja, Paloma San Basilio, La Lupe, Blanca Rosa Gil et María Félix? Ce sont toutes, à leur manière, des femmes malmenées par les épreuves de la vie, qui ont su se relever en s'affirmant aux dépens des hommes. L'actrice María Félix (1914-2002) qui disait être une femme au cœur d'homme n'avait-elle pas brisé le stéréotype de la femme mexicaine de son époque, paysanne et soumise? De leur côté, la chanteuse de jazz portoricaine Lucy Fabery (1931), aussi surnommée "La Muñeca de Chocolate» en raison de la beauté de sa peau noire, la star espagnole Sara Montiel (1928) qui introduisit dans le cinéma de l'Espagne franquiste un érotisme troublant qui ferait se pâmer le Caudillo lui-même, la chanteuse cubaine La Lupe (1936-1992) et ses provocations sont autant d'exemples de séductrices-dominatrices tirant leur pouvoir d'une plastique irréprochable et d'un glamour tout hollywoodien. Envoûtantes par leur voix et leurs chansons, ces divas fatales parviennent à prendre les hommes dans leurs griffes et à affirmer leur place et leur revanche sociale. On ne sera pas surpris de constater que Pedro Almodóvar dont on connaît l'affection pour les travestis et le goût pour le questionnement du genre n'hésite pas à rendre hommage à ces femmes, notamment La Lupe dans Mujeres al borde de un ataque de nervios et Sara Montiel dans La mala educación.

Enfin, si certaines féministes peuvent être offensées que le travesti masculin soit hypertélique, c'est-à-dire qu'il grossisse jusqu'à la caricature les traits supposés de la féminité, des érotiques aux psychologiques, il faut bien se rendre compte que, quand bien même le travesti imite la femme, il «ne cherche pas à rendre sa féminité croyable» (Zapata 2008: 525). Il cherche, pour reprendre le texte de Máscaras, à «sobrepasar el límite, yendo más allá de la mujer» (50) et se joue autant du féminin que du masculin. C'est une «imagen que se sabe imagen" comme l'explique le grand auteur cubain José Lezama Lima (1981: 218). Pour l'anthropologue Esther Newton dans Mother Camp. Female Impersonators in America, le travesti «est une double inversion qui dit les apparences sont trompeuses. Le drag dit mon 
apparence extérieure est féminine mais mon essence intérieure [le corps] est masculine. Au même moment, il symbolise l'inversion contraire, mon apparence extérieure [mon corps, mon genre] est masculine mais mon essence intérieure [moi-même] est féminine»(Butler 2006: 260). Déjà, en 1969, l'écrivain cubain Severo Sarduy (1969: 48), analysant El lugar sin límites du Chilien José Donoso et son protagoniste travesti Manuela, expliquait que:

lo que Manuela nos hace ver no es una mujer bajo la apariencia de la cual se escondería un hombre, una máscara cosmética que al caer dejara al descubierto una barba, un rostro ajado y duro, sino el hecho mismo del travestismo. Nadie ignora, y sería imposible ignorarlo dada la evidencia del disfraz, la nitidez del artificio, que Manuela es un ajetreado bailarín, un hombre disimulado, un capricho. Lo que Manuela muestra es la coexistencia, en un solo cuerpo, de significantes masculinos y femeninos: la tensión, la repulsión, el antagonismo que entre ellos se crea.

El Recio dans Máscaras adhère complètement à cette idée. Pour lui, il est impossible que le travesti puisse abuser complètement autrui:

la erección cosmética del travestí, [...] la agresión resplandeciente de sus párpados temblorosos y metalizados como alas de insectos voraces, su voz desplazada, como si perteneciera a otro personaje, siempre en off, la boca pretendida, dibujada sobre su boca escondida, y su propio sexo, más presente cuanto más castrado, es todo una apariencia, algo así como una perfecta mascarada teatral. (48-49).

On est dans le simulacre dont nous parle Baudrillard, c'est-à-dire un système de signes sans référent extralinguistique dans lequel un signe nous conduit à un autre et où la réalité n'existe pas hors du simulacre (Dorado Otero 2008). Il y a des détails qui ne trompent pas comme « una nuez de Adán inevitable, unas manos crecidas por designio natural, una pelvis estrecha, ajena a cualquier atisbo de maternidad...» (Padura Fuentes 1997: 74). De fait, dans le roman de Pedro Juan Gutiérrez, Rey ne se laisse pas tromper et reconnaît l'entre-deux qu'est Sandra, "una mujer bellísima, pero al mismo tiempo un hombre bellísimo» (63), ce qui l'excite fortement (89-90) ${ }^{6}$. Néanmoins, Mario Conde, dans Máscaras, n'est pas aussi convaincu que les travestis ne peuvent pas abuser autrui, ce qui ne manque pas de dérouter le macho

6 Dans Le théorème d'Almodóvar (2008: 73-74), l'auteur français, d'origine catalane, Antoni Casas Ros (1972) explique combien un homme travesti en femme peut être excitant pour des hommes hétérosexuels: "Non, moi j'aime bien être actif et beaucoup d'hommes recherchent ça. Ils sont attirés par la sodomie mais pas assez pour la tenter avec un homme. Avec nous la peur tombe. Ils ne se sentent pas homos. Ils voient un visage de femme, un corps de femme et ils sentent quelque chose qui les pénètre. Une autre demande. Plus ambiguë». 
qu'il est: «de no estar advertido, la habría considerado [Viki] una mujer si no hermosa, al menos apetecible» (145) 7 . Il se met d'ailleurs à douter de l'«authenticité» de toutes les femmes qu'il croise (141/230)!

La plupart des personnages qui apparaissent dans Conversaciones con Aurelia sont présentés comme des travestis peut-être en raison du spectacle, de la performance, qu'ils sont amenés à produire. De fait, les vêtements mais surtout les longues séances de maquillage essayent de cacher les « vestigios de masculinidad» (20) sur des visages présentés comme des paysages au relief délateur, précisément réorganisés par les mains expertes de cantonniers se rebellant contre la nature ${ }^{8}$. Pourtant, dans le roman, il semble que les personnages aient plutôt une identité transsexuelle mais ils n'ont pas encore franchi le pas de la transformation complète, de la mise en adéquation entre ce qu'ils sont intérieurement et leur corps ${ }^{9}$. Presque tous les personnages ont des seins siliconés et s'injectent des hormones mais leur silhouette, la longueur de leurs mains, leur pilosité continuent de les trahir (82): «Todavía no habías llegado al punto álgido, en el que fueras lluvia de femeninos en el cuerpo, mujer total sin desvelos de ninguna clase» (83), lance la voix narrative à Nani qui imite la chanteuse espagnole Paloma San Basilio. Dans El Rey de la Habana, Sandra a aussi une identité transsexuelle qu'elle aimerait bien concrétiser par une opération, trop coûteuse malheureusement:

[parlant de son sexe et de ses testicules] Si yo pudiera, me los cortaba. ¡No quiero ser hombre! Lo que más quiero en la vida es ser mujer. Una mujer normal. Con todo. Con una vagina húmeda y olorosa y dos pechos grandes y hermosos y un buen culón, y tener un marido que me quiera y me cuide, y me preñe, y parirle tres o cuatro hijos. (91)

En attendant, elle a commencé son processus de transformation en s'injectant de la Medrone, "una pastilla anticonceptiva y reguladora de la menstruación, a base de hormonas femeninas» (65) qui lui fait pousser

7 Le Marquis partage cette idée: «aquella mujer hubiera arrebatado a cualquier hombre porque era más perfecta que una mujer, casi diría que era la mujer» (48).

8 «Te estabas mirando en el espejo. Tu rostro era como un papel en blanco en el que trazabas varias líneas. Organizaste los colores uno junto al otro, en fila, antes de comenzar el elaborado maquillaje que constituiría tu único modo de ser en el escenario: donde la naturaleza se transformaba en artificio» (13). Lire aussi ce passage de El Rey de la Habana: «Sandra, en blúmers y con las teticas al aire, comenzó a maquillarse. Primero se rasuró bien la cara, las axilas, las piernas. Cremas suavizadoras, bases, polvos, pintura de labios, peluca rubia, sombra en los ojos, pestañas postizas, uñas postizas. Le llevó más de una hora. Aquel mulato hermoso, andrógino, bello, fue mutando lentamente en una mulata especialmente atractiva, con un fuerte magnetismo sexual». (77).

9 Pour mieux saisir la diversité des parcours et profils au sein du monde du spectacle, Maxime Foerster (2006: 87) parle de «cabaret transgenre». 
les seins et qui lui permet de se convaincre que, entre elle et les "vraies» femmes, il n'y a pas de différence (64-65).

Pourtant, des différences il semble y en avoir et, loin des lumières artificielles du cabaret, les transsexuels sont souvent victimes d'insultes transphobes comme Delirio, dans Conversaciones con Aurelia, a pu l'expérimenter en se rendant en bus de Caguas à San Juan (65), non pas entre chien et loup mais en plein jour. Après les sifflements moqueurs des hommes présents au centre de bus, Delirio doit affronter les commentaires haineux de doña Calixta, « una hermana de la iglesia Pentescostal» (66) qui, non contente de se plaindre de la présence de Delirio dans le bus ${ }^{10}$, se met à lire la Bible y «a predicar en grande dando todo un señor sermón, con coritos intercalados, sobre los degenerados sexuales» (68). Cet épisode éprouvant pour Delirio nous est pourtant conté avec humour. Le comportement de doña Calixta est comparé à deux reprises à une performance et son portrait physique ferait presque douter qu'il s'agisse vraiment d'une femme:

Mediste su moño canoso recogido hacia atrás escondiendo una cabellera que le llegaría hasta las nalgas caídas, miraste el reguero de arrugas de su cara de dama consagrada a la obra del Señor, te detuviste en sus rodillas peludas que auguraban unas piernas bien torneadas pero cubiertas de vello femenino. (66-67)

Finalement, Delirio incarne plus l'image, non pas l'essence, féminine! Quant à la performance de doña Calixta, elle se solde par un échec dans la mesure où la plupart des usagers du bus critiquent l'Eglise qui se mêle de la vie des autres (68). Delirio ne rentre pas dans le jeu de la nonne et se contente d'incliner délicatement la tête et s'évade par la pensée en s'enivrant dans l'amour tout aussi biblique que dégagent David et Jonathan ou Ruth et Nohemi (66). D'autres transsexuels, en revanche, ont une attitude plus revendicatrice - que certains esprits grincheux pourraient qualifier de provocante - et se plaisent à jouer avec l'ambiguïté:

La ambigüedad de no saberse si era macho o hembra a ciencia cierta. Entrar a un mall de Columbus o Cincinnati a comprar ropa de mujer vestido de calle sin maquillaje ni uñas ni carterón ni tacas altas. Con su mahoncito y su camisetita color rosita vivo, demasiado ajustada para mostrar su tetamen silicónico, que contradecía esa cara lavada y esas manos demasiado grandes para ser mujer. Siempre le encantaba equilibrarse como trapecista en la cuerda floja del qué dirán. (76).

10 «De qué barbaridad, cómo estaba el mundo porque la poca vergüenza ahora se atrevía a salir a la calle» (66). 
En cela, ils sont proches destransgenres, des personnes dont l'identité de genre est en conflit avec celle traditionnellement attribuée aux personnes de même sexe. Cela rappelle la transsexualité à la différence près que les transgenres sont dans une dynamique très différente de celle des personnes transsexuelles car ils n'entreprennent pas (et ne veulent surtout pas entreprendre) d'opération de réattribution de sexe (Eribon 2003: 472). En France, comme le rappelle Maxime Foerster (2006: 157), le terme trans', avec une apostrophe à la fin, "remplace de plus en plus transsexuel et transgenre pour rendre compte d'une irréductible diversité de parcours, de genres et d'horizons d'attente».

Dans Conversaciones con Aurelia, avec Nani, seule Meche est allée au bout du processus opératoire afin de mettre en conformité totale son identité et son corps, son sexe anatomique et son sexe psychologique. Elle est enfin telle qu'elle aurait dû être mise au monde: «hecha toda mujer delicada de veras» (72). Même dotée d'une «vagina de quirófano» (30), l'opération lui a conféré la qualité recherchée de véritable «fémina de las crueles y fatales " (30). On notera le jeu sur le genre grammatical qui témoigne de ce passage, masculin avant l'opération, féminin ensuite:

Acostado, cuan largo eras, sobre la mesa de operaciones, te hundías en los algodones de la anestesia hasta perder toda noción del tiempo, entre los cortes, la mutilación, la reconstrucción de tu cuerpo dejándote vano como un sueño flotando en medio de los azules que había por todas partes, como otro papel en blanco. Y fueron tomando lentamente los instrumentos de la bandeja donde estaban dispuestos uno al lado del otro, en fila, para irlos regresando con golpes secos a otra bandeja ya manchados de sangre y listos para ser esterilizados una vez más. Tú, en cambio, quedaste recostada sobre la mesa de operaciones, convertid $a$ ya, lind $a$ y mimosa, en los ecos de unas largas conversaciones con Aurelia, Gran Madama del Dulce de Coco. (63)

L'opération n'est pas sans susciter la jalousie des camarades du cabaret qui dénient alors à Meche tout talent artistique dans la mesure où elle n'a pas à faire le moindre effort pour imiter la femme (18). Dans le discours de Delirio, on comprend que la transsexualité est normative. De fait, pour reprendre les propos de Natacha Taurisson, la transsexualité, "c'est en quelque sorte remettre en ordre et en équilibre l'être humain concerné» (Taurisson \& Marzano 2005: 103). La transsexualité ne vise pas à abolir les genres, ni à jouer avec eux, mais à acquérir le genre que l'on a intérieurement et qui n'est pas marqué physiquement et se place dans un conformisme radical. Le discours de Sandra, précédemment cité, dans lequel elle déclare désirer une vie rangée, est, à cet égard, particulièrement éclairant. 
En conclusion, il est intéressant de souligner que les personnages qui apparaissent dans nos trois romans sont pratiquement tous désignés comme étant des travestis alors même que leur identité est transsexuelle. Cela s'explique par la méconnaissance des différences entre le simple travestissement et la transsexualité même s'il existe « des formes de passage » entre eux (Esturgie 2008: 50), renforcée par le fait que la plupart des personnages sont des performeurs qui travaillent dans des cabarets et qu'ils n'ont pas subis d'opérations de réassignation sexuelle. Si le travesti et le transsexuel partagent d'évidents traits communs, la démarche de chacun est bien différente. Dans un cadre politique, si le premier met à nu le caractère construit des normes sexuelles élargissant de la sorte la sphère des êtres sexuels légitimes, le second ne désire pas autre chose que se lover du bon côté de la douce binarité homme / femme et ainsi, pour reprendre les paroles de la chanteuse Juliette dans Garçon manqué (2002), réparer «la perpétuelle punition, la cruelle contrefaçon, l'erreur de fabrication ».

\section{Bibliographie}

Balutet, Nicolas, 2004, «Mi madre en vez de leche me dio sometimiento. De la condition féminine dans Balún Canán", in Néstor Ponce, Ecrire la domination en Amérique latine (Balún Canán de Rosario Castellanos, Los ríos profundos de José María Arguedas, El chulla Romero y Flores de Jorge Icaza), Nantes, Editions du Temps, p. 77-88.

Balutet, Nicolas, 2008, Homosexualité et imaginaire sexuel chez les Aztèques, Oxford, Archaeopress.

Butler, Judith, 2006, Trouble dans le genre. Le féminisme et la subversion de l'identité, Paris, La Découverte.

Casas Ros, Antoni, 2008, Le théorème d'Almodóvar, Paris, Gallimard.

Dorado Otero, Angela, sous presse, «Las fisuras del sistema: el travestismo como medio discursivo de subversión del hombre nuevo en la monología estatal cubana ", in Image et manipulation. Actes du $6^{e}$ Congrès International du GRIMH, Lyon, GRIMH / LCE.

Eribon, Didier, 2003, «Transgenres», in Didier Eribon, Dictionnaire des cultures gays et lesbiennes, Paris, Larousse, p. 472.

Esturgie, Claude, 2008, Le genre en question ou questions de genre. De Pierre Molinier à Pedro Almodóvar, Paris, Editions Léo Scheer.

Foerster, Maxime, 2006, Histoire des transsexuels en France, Béziers, H\&O Editions.

Garber, Marjorie, 1992, Vested Interests. Cross-dressing and Cultural Anxiety, New York, Routledge. 
Gutiérrez, Pedro Juan, 1999, El Rey de la Habana, Barcelona, Anagrama.

La Cecla, Franco, 2002, Ce qui fait un homme, Paris, Liana Levi.

Lezama Lima, José, 1981, El reino de la imagen, Caracas, Biblioteca Ayacucho.

Ortner, Sherry, 1972, "Is Female to Male as Nature is to Culture? », Feminist Studies, $\mathrm{n}^{\circ} 1,1972$, p. 5-31.

Padura Fuentes, Leonardo, 1997, Máscaras, Tusquets, Barcelona.

Sarduy, Severo, 1969, "Escritura/travestismo", in Severo Sarduy, Escrito sobre un cuerpo. Ensayos de crítica, Buenos Aires, Editorial Sudamericana, p. 46-48.

Taurisson, Natacha et Michela Marzano, 2005, «Transsexualisme, corps et changement d'identité», Cités, n² 21, p. 103-112.

Tcherkaski, José, 1998, Habla Copi. Homosexualidad y creación, Buenos Aires, Galerna.

Torres, Daniel, 2007, Conversaciones con Aurelia, Puerto Rico, Isla Negra Editores.

Welzer-Lang, Daniel, 2004, Les hommes aussi changent, Paris, Payot.

Zapata, Mónica, 2008, "Performance du genre et fabrique du corps", in Image et corps. Actes du $5^{e}$ Congrès International du GRIMH. Hommage à Emmanuel Larraz, Lyon, GRIMH / LCE, p. 523-533. 